\title{
153.
}

\section{A MEMOIR ON THE AUTOMORPHIC LINEAR TRANSFORMATION OF A BIPARTITE QUADRIC FUNCTION.}

[From the Philosophical Transactions of the Royal Society of London, vol. CxLviII. for the year 1858, pp. 39-46. Received December 10, 1857,-Read January 14, 1858.]

THE question of the automorphic linear transformation of the function $x^{2}+y^{2}+z^{2}$, that is the transformation by linear substitutions, of this function into a function $x_{1}^{2}+y_{1}^{2}+z_{1}^{2}$ of the same form, is in effect solved by some formulæ of Euler's for the transformation of coordinates, and it was by these formulæ that I was led to the solution in the case of the sum of $n$ squares, given in my paper "Sur quelques propriétés des déterminants gauches" $\left.{ }^{1}\right)$. A solution grounded upon an à priori investigation and for the case of any quadric function of $n$ variables, was first obtained by M. Hermite in the memoir "Remarques sur une Mémoire de M. Cayley relatif aux déterminants gauches" $\left.{ }^{2}\right)$. This solution is in my Memoir "Sur la transformation d'une function quadratique en elle-même par des substitutions linéaires" $\left.{ }^{3}\right)$, presented under a somewhat different form involving the notation of matrices. I have since found that there is a like transformation of a bipartite quadric function, that is a lineo-linear function of two distinct sets, each of the same number of variables, and the development of the transformation is the subject of the present memoir.

1. For convenience, the number of variables is in the analytical formulæ taken to be 3 , but it will be at once obvious that the formulæ apply to any number of variables whatever. Consider the bipartite quadric

$$
\left(\begin{array}{lll}
a, & b, & c \\
a^{\prime}, & b^{\prime}, & c^{\prime} \\
a^{\prime \prime}, & b^{\prime \prime}, & c^{\prime \prime}
\end{array} \mid\right.
$$

${ }_{1}$ Crelle, t. xxxir. (1846) pp. 119-123, [52].

${ }^{2}$ Cambridge and Dublin Mathematical Journal, t. 1x. (1854) pp, 63-67.

3 Crelle, t. L. (1855) pp. 288-299, [136].

C. II. 
which stands for

$$
\begin{array}{r}
(a x+b y+c z) \mathrm{x} \\
+\left(a^{\prime} x+b^{\prime} y+c^{\prime} z\right) \mathrm{y} \\
+\left(a^{\prime \prime} x+b^{\prime \prime} y+c^{\prime \prime} z\right) \mathrm{z}
\end{array}
$$

and in which $(x, y, z)$ are said to be the nearer variables, and $(\mathrm{x}, \mathrm{y}, \mathrm{z})$ the further variables of the bipartite.

2. It is clear that we have

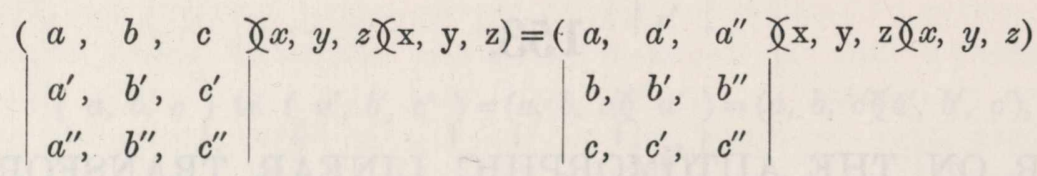

and the new form on the right-hand side of the equation may also be written

$$
\left(\operatorname{tr} .\left(\begin{array}{lll}
a, & b, & c
\end{array}\right) \gamma \mathrm{x}, \mathrm{y}, \mathrm{z} \chi x, y, z\right),
$$

that is, the two sets of variables may be interchanged, provided that the matrix is transposed.

3. Each set of variables may be linearly transformed: suppose that the substitutions are

and

$$
(x, y, z)=\left(\begin{array}{lll}
l, & m, & \bar{n} \\
l^{\prime}, & m^{\prime}, & n^{\prime} \\
l^{\prime \prime}, & m^{\prime \prime}, & y_{1}^{\prime \prime}
\end{array} \mid z_{l}\right)
$$

$$
(x, y, z)=\left(\begin{array}{lll}
l, & l^{\prime}, & l^{\prime \prime} \\
m, & m^{\prime}, & m^{\prime \prime} \\
n, & n^{\prime}, & n_{1}^{\prime \prime}
\end{array} \mid\right.
$$

Then first substituting for $(x, y, z)$ their values in terms of $\left(x, y_{1}, z_{l}\right)$, the bipartite becomes

$$
\left(\left(\begin{array}{lll}
a, & b, & c \\
a^{\prime}, & b^{\prime}, & c^{\prime} \\
a^{\prime \prime}, & b^{\prime \prime}, & c^{\prime \prime}
\end{array}|| \begin{array}{lll}
l, & m, & n \\
l^{\prime}, & m^{\prime}, & n^{\prime} \\
l^{\prime \prime}, & m^{\prime \prime}, & n^{\prime \prime}
\end{array} \mid \gamma\left(x_{1}, y_{1}, z, \gamma \mathrm{x}, \mathrm{y}, \mathrm{z}\right)\right.\right.
$$

represent for a moment this expression by

$$
\left(\begin{array}{lll}
A, & B, & C \\
A^{\prime}, & B^{\prime}, & C^{\prime} \\
A^{\prime \prime}, & B^{\prime \prime}, & C^{\prime \prime}
\end{array} \mid\right.
$$


then substituting for $(x, y, z)$ their values in terms of $\left(x_{1}, y_{1}, z_{l}\right)$, it is easy to see that the expression becomes

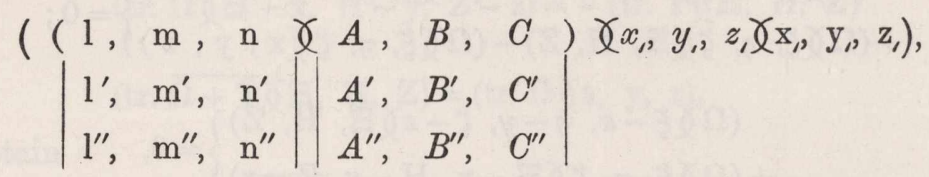

and re-establishing the value of the auxiliary matrix, we obtain, as the final result of the substitutions,

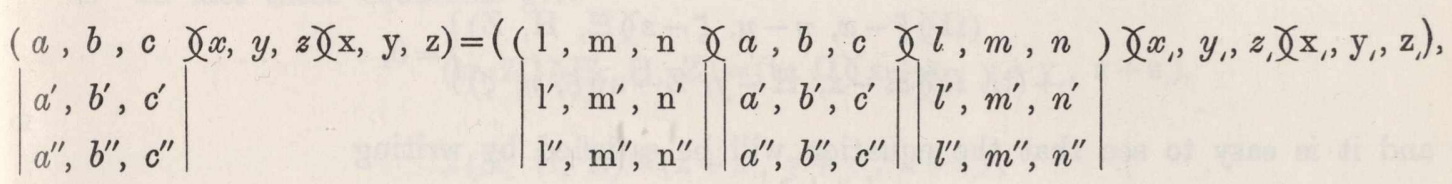

that is, the matrix of the transformed bipartite is obtained by compounding in order, first or furthest the transposed matrix of substitution of the further variables, next the matrix of the bipartite, and last or nearest the matrix of substitution of the nearer variables.

4. Suppose now that it is required to find the automorphic linear transformation of the bipartite

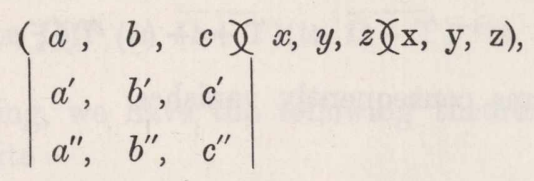

or as it will henceforward for shortness be written,

$$
(\Omega \gamma x, y, z \gamma \mathrm{x}, \mathrm{y}, \mathrm{z}) \text {; }
$$

this may be effected by a method precisely similar to that employed by M. Hermite for an ordinary quadric. For this purpose write

$$
\begin{array}{lll}
x+x_{1}=2 \xi, & y+y_{1}=2 \eta, & z+z_{1}=2 \zeta, \\
\mathrm{x}+\mathrm{x},=2 \Xi, & \mathrm{y}+\mathrm{y}_{1}=2 \mathrm{H}, & \mathrm{z}+\mathrm{z}_{\imath}=2 \mathrm{Z},
\end{array}
$$

or, as these equations may be represented,

then we ought to have

$$
\begin{aligned}
& \left(x+x, \quad y+y_{1}, \quad z+z\right)=2(\dot{\xi}, \eta, \zeta), \\
& \left(\mathrm{x}+\mathrm{x}_{\imath}, \mathrm{y}+\mathrm{y}_{\imath}, \mathrm{z}+\mathrm{z}\right)=2(\Xi, \mathrm{H}, \mathrm{Z}) ;
\end{aligned}
$$

$$
(\Omega \gamma 2 \xi-x, 2 \eta-y, 2 \zeta-z \chi 2 \Xi-x, 2 \mathrm{H}-\mathrm{y}, 2 \mathrm{Z}-\mathrm{z})=(\Omega \gamma(x, y, z \gamma \mathrm{x}, \mathrm{y}, \mathrm{z}) .
$$

5. The left-hand side is

$4(\Omega \gamma \xi, \eta, \zeta \gamma \equiv, \mathrm{H}, \mathrm{Z})-2(\Omega \gamma(x, y, z \chi \Xi, \mathrm{H}, \mathrm{Z})-2(\Omega \gamma \xi, \eta, \zeta \gamma \mathrm{x}, \mathrm{y}, \mathrm{z})+\Omega \gamma(x, y, z \gamma \mathrm{x}, \mathrm{y}, \mathrm{z})$, and the equation becomes

$$
2(\Omega \gamma \xi, \eta, \zeta \gamma \equiv, \mathrm{H}, \mathrm{Z})-(\Omega \chi x, y, z \chi \Xi, \mathrm{H}, \mathrm{Z})-(\Omega \chi \xi, \eta, \zeta \gamma \mathrm{x}, \mathrm{y}, \mathrm{z})=0,
$$


or as it may be written,

or again,

$$
\left.\begin{array}{r}
(\Omega \gamma \xi, \eta, \zeta \gamma \equiv, \mathrm{H}, \mathrm{Z})-(\Omega \gamma x, y, z \gamma \equiv, \mathrm{H}, \mathrm{Z}) \\
+(\Omega \gamma \xi, \eta, \zeta \gamma \equiv, \mathrm{H}, \mathrm{Z})-(\Omega \gamma \xi, \eta, \zeta \gamma \mathrm{\gamma}, \mathrm{y}, \mathrm{z})
\end{array}\right\}=0 ;
$$

$$
\left.\begin{array}{r}
(\Omega \gamma \xi-x, \eta-y, \zeta-z \gamma \Xi, \mathrm{H}, \mathrm{Z}) \\
+(\Omega \gamma \xi, \eta, \zeta \gamma \Xi-\mathrm{x}, \mathrm{H}-\mathrm{y}, \mathrm{Z}-\mathrm{z})
\end{array}\right\}=0,
$$

or what is the same thing,

$$
\left.\begin{array}{r}
(\Omega \gamma \xi-x, \eta-y, \zeta-z \gamma \Xi, \mathrm{H}, \mathrm{Z}) \\
+(\operatorname{tr} . \Omega \gamma \Xi-\mathrm{x}, \mathrm{H}-\mathrm{y}, \mathrm{Z}-\mathrm{z} \gamma \xi, \eta, \zeta)
\end{array}\right\}=0,
$$

and it is easy to see that the equation will be satisfied by writing

$$
\begin{aligned}
& (\Omega \gamma \xi-x, \eta-y, \zeta-z)=\left(\Upsilon_{\gamma} \xi, \eta, \zeta\right) \text {, } \\
& (\operatorname{tr} . \Omega \gamma \Xi-\mathrm{x}, \mathrm{H}-\mathrm{y}, \mathrm{Z}-\mathrm{z})=-\left(\operatorname{tr} . \Upsilon \Upsilon^{\prime} \Xi, \mathrm{H}, \mathrm{Z}\right) \text {, }
\end{aligned}
$$

where $\Upsilon$ is any arbitrary :matrix. In fact we have then

$$
\begin{aligned}
& (\Omega \gamma \xi-x, \eta-y, \zeta-z \gamma \Xi, \mathrm{H}, \mathrm{Z})=\left(\Upsilon_{\gamma} \boldsymbol{\gamma}, \eta, \zeta \gamma \Xi, \mathrm{H}, \mathrm{Z}\right) \text {, } \\
& (\operatorname{tr} . \Omega \gamma \Xi-\mathrm{x}, \mathrm{H}-\mathrm{y}, \mathrm{Z}-\mathrm{z} \gamma \xi, \eta, \zeta)=-\left(\operatorname{tr} . \Upsilon_{\chi} \boldsymbol{\gamma} \Xi, \mathrm{H}, \mathrm{z} \gamma \xi, \eta, \zeta\right) \\
& =-(\Upsilon \gamma \xi, \eta, \zeta \gamma \equiv, H, Z),
\end{aligned}
$$

and the sum of the two terms consequently vanishes.

6. The equation

gives

$$
(\Omega \gamma \xi-x, \eta-y, \zeta-z)=(\Upsilon \gamma \xi, \eta, \zeta)
$$

$$
(\Omega-\Upsilon \gamma \xi, \eta, \zeta)=(\Omega \curlyvee x, y, z),
$$

and we then have

$$
(\Omega+\Upsilon \curlyvee \xi, \eta, \zeta)=\left(\Omega \curlyvee x, y_{1}, z\right) .
$$

In fact the two equations give

$$
(2 \Omega \gamma \xi, \eta, \zeta)=\left(\Omega \gamma\left(x+x, y+y_{1}, z+z\right),\right.
$$

or what is the same thing,

$$
2(\xi, \eta, \zeta)=\left(x+x, y+y_{1}, z+z_{\imath}\right),
$$

which is the equation assumed as the definition of $(\xi, \eta, \zeta)$; and conversely, this equation, combined with either of the two equations, gives the other of them.

7. We have consequently

and thence

$$
\begin{aligned}
& (x, y, z)=\left(\Omega^{-1}(\Omega-\Upsilon) \gamma \xi, \eta, \zeta\right), \\
& (\xi, \eta, \zeta)=\left((\Omega+\Upsilon)^{-1} \Omega \gamma\left(x_{1}, y_{1}, z_{1}\right),\right.
\end{aligned}
$$

$$
(x, y, z)=\left(\Omega^{-1}(\Omega-\Upsilon)(\Omega+\Upsilon)^{-1} \Omega \gamma\left(x, y_{1}, z\right) .\right.
$$


8. But in like manner the equation

$$
\text { (tr. } \Omega \curlyvee \Xi-x, H-y, Z-z)=-(\operatorname{tr} . \Upsilon \curlyvee \equiv, H, Z)
$$

gives

$$
\text { (tr. } \overline{\Omega+\Upsilon \gamma} \text { 久 } \equiv, \mathrm{H}, \mathrm{Z})=(\operatorname{tr} . \Omega \gamma \mathrm{x}, \mathrm{y}, \mathrm{z}) \text {, }
$$

and we then obtain

$$
(\operatorname{tr} . \overline{\Omega-\Upsilon} \gamma \equiv, H, Z)=\left(\operatorname{tr} . \Omega \gamma x_{i}, y_{i}, z_{i}\right) .
$$

9. In fact these equations give

$$
\begin{aligned}
& (\operatorname{tr} .2 \Omega \gamma \Xi, H, Z)=\left(\operatorname{tr} . \Omega \gamma\left(x+x_{1}, y+y_{1}, z+z_{1}\right),\right. \\
& 2(\Xi, H, Z)=\left(x+x_{1}, y+y_{1}, z+z_{\imath}\right) ;
\end{aligned}
$$

and conversely, this equation, combined with either of the two equations, gives the other of them. We have then

$$
\begin{aligned}
& (x, y, z)=\left((\operatorname{tr} . \Omega)^{-1}(\operatorname{tr} . \overline{\Omega+\Upsilon} \gamma \Xi, H, z),\right. \\
& (\Xi, H, z)=\left((\operatorname{tr} . \overline{\Omega-\Upsilon})^{-1} \operatorname{tr} . \Omega \gamma x_{1}, y_{1}, z_{)}\right),
\end{aligned}
$$

and thence

$$
(\mathrm{x}, \mathrm{y}, \mathrm{z})=\left((\operatorname{tr} . \Omega)^{-1}(\operatorname{tr} . \overline{\Omega+\Upsilon})(\operatorname{tr} . \overline{\Omega-\Upsilon})^{-1} \operatorname{tr} . \Omega_{\chi} \mathrm{x}_{l}, \mathrm{y}_{l}, \mathrm{z}_{\iota}\right) .
$$

10. Hence, recapitulating, we have the following theorem for the automorphic linear transformation of the bipartite

$$
(\Omega \mathcal{\gamma} x, y, z \gamma \mathrm{\gamma}, \mathrm{y}, \mathrm{z}),
$$

viz. $\Upsilon$ being an arbitrary matrix, if

$$
\begin{aligned}
& (x, y, z)=\left(\Omega^{-1}(\Omega-\Upsilon)(\Omega+\Upsilon)^{-1} \Omega \gamma_{x}, y_{l}, z_{l}\right), \\
& (\mathrm{x}, \mathrm{y}, \mathrm{z})=\left((\operatorname{tr} . \Omega)^{-1}(\operatorname{tr} . \overline{\Omega+\Upsilon})(\operatorname{tr} . \overline{\Omega-\Upsilon})^{-1} \operatorname{tr} . \Omega \gamma_{\left(x_{l}\right.}, y_{l}, z_{l}\right),
\end{aligned}
$$

then

$$
(\Omega \gamma x, y, z \gamma \mathrm{x}, \mathrm{y}, \mathrm{z})=\left(\Omega \gamma\left(x, y_{1}, z, \chi_{\mathrm{x}}, \mathrm{y}_{1}, \mathrm{z}\right),\right.
$$

which is the theorem in question.

11. I have thought it worth while to preserve the foregoing investigation, but the most simple demonstration is the verification $\dot{a}$ posteriori by the actual substitution of the transformed values of $(x, y, z),(\mathrm{x}, \mathrm{y}, \mathrm{z})$. To effect this, recollecting that in general tr. $\left(A^{-1}\right)=(\operatorname{tr} . A)^{-1}$ and tr. $A B C D=\operatorname{tr} . D$. tr. $C$. tr. $B$. tr. $A$, the transposed matrix of substitution for the further variables is

$$
\Omega(\Omega-\Upsilon)^{-1}(\Omega+\Upsilon) \Omega^{-1} ;
$$

and compounding this with the matrix $\Omega$ of the bipartite, and the matrix

$$
\Omega^{-1}(\Omega-\Upsilon)(\Omega+\Upsilon)^{-1} \Omega
$$


of substitution for the nearer variables, the theorem will be verified if the result is equal to the matrix $\Omega$ of the bipartite; that is, we ought to have

$$
\Omega(\Omega-\Upsilon)^{-1}(\Omega+\Upsilon) \Omega^{-1} \Omega \Omega^{-1}(\Omega-\Upsilon)(\Omega+\Upsilon)^{-1} \Omega=\Omega,
$$

or what is the same thing,

$$
\Omega(\Omega-\Upsilon)^{-1}(\Omega+\Upsilon) \Omega^{-1}(\Omega-\Upsilon)(\Omega+\Upsilon)^{-1} \Omega=\Omega ;
$$

this is successively reducible to

$$
\begin{aligned}
& (\Omega+\Upsilon) \Omega^{-1}(\Omega-\Upsilon)=(\Omega-\Upsilon) \Omega^{-1}(\Omega+\Upsilon) \\
& \Omega^{-1}(\Omega+\Upsilon) \Omega^{-1}(\Omega-\Upsilon)=\Omega^{-1}(\Omega-\Upsilon) \Omega^{-1}(\Omega+\Upsilon) \\
& \left(1+\Omega^{-1} \Upsilon\right)\left(1-\Omega^{-1} \Upsilon\right)=\left(1-\Omega^{-1} \Upsilon\right)\left(1+\Omega^{-1} \Upsilon\right)
\end{aligned}
$$

which is a mere identity, and the theorem is thus shown to be true.

12. It is to be observed that, in the general theorem, the transformations or matrices of substitution for the two sets of variables respectively are not identical, but it may be required that this shall be so. Consider first the case where the matrix $\Omega$ is symmetrical, the necessary condition is that the matrix $\Upsilon$ shall be skew symmetrical; in fact we have then

$$
\text { tr. } \Omega=\Omega, \text { tr. } \Upsilon=-\Upsilon,
$$

and the transformations become

$$
\begin{aligned}
& (x, y, z)=\left(\Omega^{-1}(\Omega-\Upsilon)(\Omega+\Upsilon)^{-1} \Omega \gamma\left(x, y_{l}, z_{l}\right),\right. \\
& (x, y, z)=\left(\Omega^{-1}(\Omega-\Upsilon)(\Omega+\Upsilon)^{-1} \Omega \gamma x_{i}, y_{1}, z_{l}\right),
\end{aligned}
$$

which are identical. We may in this case suppose that the two sets of variables become equal, and we have then the theorem for the automorphic linear transformation of the ordinary quadric

$$
(\Omega \gamma x, y, z)^{2},
$$

viz. $\Upsilon$ being a skew symmetrical matrix, if

then

$$
(x, y, z)=\left(\Omega^{-1}(\Omega-\Upsilon)(\Omega+\Upsilon)^{-1} \Omega \gamma\left(x, y, z_{l}\right),\right.
$$

$$
\left(\Omega \gamma(x, y, z)^{2}=\left(\Omega \gamma\left(x, y_{1}, z\right)^{2} .\right.\right.
$$

13. Next, if the matrix $\Omega$ be skew symmetrical, the condition is that the matrix $\Upsilon$ shall be symmetrical; we have in this case tr. $\Omega=-\Omega$, tr. $\Upsilon=\Upsilon$, and the four factors in the matrix of substitution for $(\mathrm{x}, \mathrm{y}, \mathrm{z})$ are respectively $-\Omega^{-1},-(\Omega-\Upsilon),-(\Omega+\Upsilon)^{-1}$ and $-\Omega$, and such matrix of substitution becomes therefore, as before, identical with that for $(x, y, z)$; we have therefore the following theorem for the automorphic linear transformation of a skew symmetrical bipartite

$$
(\Omega \gamma(x, y, z \gamma \mathrm{x}, \mathrm{y}, \mathrm{z}),
$$


when the transformations for the two sets of variables are identical, viz. $\Upsilon$ being any symmetrical matrix, if

then

$$
\begin{aligned}
& (x, y, z)=\left(\Omega^{-1}(\Omega-\Upsilon)(\Omega+\Upsilon)^{-1} \Omega \gamma\left(x_{1}, y_{1}, z\right),\right. \\
& (x, y, z)=\left(\Omega^{-1}(\Omega-\Upsilon)(\Omega+\Upsilon)^{-1} \Omega \gamma\left(x_{1}, y_{1}, z_{l}\right),\right.
\end{aligned}
$$

$$
(\Omega \gamma x, y, z \gamma \mathrm{x}, \mathrm{y}, \mathrm{z})=\left(\Omega \gamma_{\chi} x, y_{1}, \mathrm{z}_{1} \gamma_{(\mathrm{x}}, \mathrm{y}_{1}, \mathrm{z}_{\imath}\right) .
$$

14. Lastly, in the general case where the matrix $\Omega$ is anything whatever, the condition is

$$
\Omega^{-1} \Upsilon=-(\operatorname{tr} . \Omega)^{-1} \text { tr. } \Upsilon
$$

for assuming this equation, then first

$$
\Omega^{-1}(\Omega-\Upsilon)=(\operatorname{tr} . \Omega)^{-1}(\operatorname{tr} . \overline{\Omega+\Upsilon}),
$$

and in like manner

$$
\Omega^{-1}(\Omega+\Upsilon)=(\operatorname{tr} . \Omega)^{-1}(\operatorname{tr} . \overline{\Omega-\Upsilon})
$$

But we have

$$
1=(\operatorname{tr} . \Omega)^{-1}(\operatorname{tr} . \overline{\Omega-\Upsilon})(\operatorname{tr} . \overline{\Omega-\Upsilon})^{-1} \operatorname{tr} . \Omega,
$$

and therefore, secondly,

$$
(\Omega+\Upsilon)^{-1} \Omega=(\operatorname{tr} . \overline{\Omega-\Upsilon})^{-1} \operatorname{tr} . \Omega
$$

and thence

$$
\Omega^{-1}(\Omega-\Upsilon)(\Omega+\Upsilon)^{-1} \Omega=(\operatorname{tr} . \Omega)^{-1}(\operatorname{tr} . \overline{\Omega+\Upsilon})(\operatorname{tr} . \overline{\Omega-\Upsilon})^{-1} \operatorname{tr} . \Omega,
$$

or the two transformations are identical.

15. To further develope this result, let $\Omega^{-1}$ be expressed as the sum of a symmetrical matrix $Q_{0}$ and a skew symmetrical matrix $Q_{1}$, and let $\Upsilon$ be expressed in like manner as the sum of a symmetrical matrix $\Upsilon_{0}$ and a skew symmetrical matrix $r_{\text {, }}$ We have then

$$
\begin{array}{ll}
\Omega^{-1} & =Q_{0}+Q_{\prime}, \\
(\operatorname{tr} . \Omega)^{-1}=\operatorname{tr} .\left(\Omega^{-1}\right) & =Q_{0}-Q_{\prime}, \\
\Upsilon & =\Upsilon_{0}+\Upsilon_{\prime}, \\
\operatorname{tr} \Upsilon & =\Upsilon_{0}-\Upsilon_{\prime},
\end{array}
$$

and the condition, $\Omega^{-1} \Upsilon=-(\operatorname{tr} . \Omega)^{-1} \operatorname{tr} . \Upsilon$, becomes

$$
\left(Q_{0}+Q_{1}\right)\left(\Upsilon_{0}+\Upsilon_{1}\right)=-\left(Q_{0}-Q_{1}\right)\left(\Upsilon_{0}-\Upsilon_{1}\right),
$$

that is,

and we have

$$
Q_{0} \Upsilon_{0}+Q, \Upsilon=0
$$

$$
\Upsilon_{0}=-Q_{0}^{-1} Q, \Upsilon_{1}
$$


or as we may write it,

and thence

$$
\Upsilon_{0}=-\left(\frac{1}{2}\left\{\Omega^{-1}+\operatorname{tr} . \Omega^{-1}\right\}\right)^{-1}\left(\frac{1}{2}\left\{\Omega^{-1}-\operatorname{tr} . \Omega^{-1}\right\}\right) \Upsilon_{,}
$$

$$
\Upsilon=-\left(\frac{1}{2}\left\{\Omega^{-1}+\operatorname{tr} . \Omega^{-1}\right\}\right)^{-1}\left(\frac{1}{2}\left\{\Omega^{-1}-\operatorname{tr} . \Omega^{-1}\right\}\right) \Upsilon,+\Upsilon_{\prime},
$$

where $\Upsilon$, is an arbitrary skew symmetrical matrix.

16. This includes the before-mentioned special cases; first, if $\Omega$ is symmetrical, then we have simply $\Upsilon=\Upsilon$, an arbitrary skew symmetrical matrix, which is right. Next, if $\Omega$ is skew symmetrical, then $\Upsilon=-0^{-1} \Omega^{-1} \Upsilon,+\Upsilon$, which can only be finite for $\Upsilon$, $=0$, that is, we have $\Upsilon=-0^{-1} \Omega^{-1}$, and (the first part of $\Upsilon$ being always symmetrical) this represents an arbitrary symmetrical matrix. The mode in which this happens will be best seen by an example. Suppose

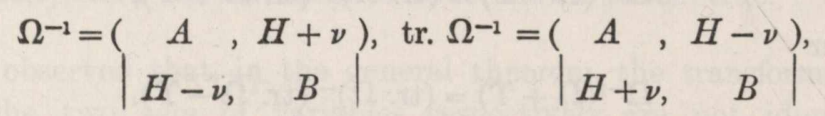

and write

then we have

$$
\Upsilon_{1}=\left(\begin{array}{rr}
0, & \theta \\
-\theta, & 0
\end{array} \mid\right.
$$

$$
\begin{aligned}
\Upsilon & =-\left(\begin{array}{ll}
A, H \\
H, & B
\end{array}|| \begin{array}{rr}
0, \nu \\
-\nu, & 0
\end{array}|| \begin{array}{rr}
0, & \theta \\
-\theta, & 0
\end{array} \mid+\left(\begin{array}{rr}
0, & \theta \\
-\theta, & 0
\end{array} \mid\right.\right. \\
& =-\frac{\nu \theta}{A B-H^{2}}\left(\begin{array}{rr}
-B, & H \\
H, & -A
\end{array} \mid+\left(\begin{array}{rr}
0, & \theta \\
-\theta, & 0
\end{array} \mid\right.\right. \\
& =\left(\begin{array}{cc}
\frac{\nu B \theta}{A B-H^{2}}, & \frac{-\nu H \theta}{A B-H^{2}}+\theta \\
\frac{-\nu H \theta}{A B-H^{2}}-\theta, & \frac{\nu A \theta}{A B-H^{2}}
\end{array} \mid+\left(\begin{array}{rr}
0, & \theta \\
-\theta, & 0
\end{array} \mid\right.\right.
\end{aligned}
$$

When $\Omega$ is skew symmetrical, $A, B, H$ vanish; but since their ratios remain arbitrary, we may write $\kappa A, \kappa B, \kappa H$ for $A, B, H$, and assume ultimately $\kappa=0$. Writing $\kappa \theta$ in the place of $\theta$, and then putting $\kappa=0$, the matrix becomes

$$
\left(\begin{array}{cc}
\frac{\nu B \theta}{A B-H^{2}}, & \frac{-\nu B \theta}{A B-H^{2}} \\
\frac{-\nu H \theta}{A B-H^{2}}, & \frac{\nu A \theta}{A B-H^{2}}
\end{array}\right)
$$

which, inasmuch as $A: \theta, B: \theta$, and $C: \theta$ remain arbitrary, represents, as it should do, an arbitrary symmetrical matrix. 
17. Hence, finally, we have the following Theorem for the automorphic linear transformation of the bipartite quadric,

$$
(\Omega \gamma(x, y, z \gamma \mathrm{x}, \mathrm{y}, \mathrm{z}),
$$

when the two transformations are identical, viz. if $\Upsilon$, be a skew symmetrical matrix, and if

then if

$$
\Upsilon=-\left(\frac{1}{2}\left\{\Omega^{-1}+\operatorname{tr} . \Omega^{-1}\right\}\right)\left(\frac{1}{2}\left\{\Omega^{-1}-\operatorname{tr} . \Omega^{-1}\right\}\right) \Upsilon,+\Upsilon_{,}
$$

$$
\begin{aligned}
& (x, y, z)=\left(\Omega^{-1}(\Omega-\Upsilon)(\Omega+\Upsilon)^{-1} \Omega \gamma\left(x, y_{1}, z\right),\right. \\
& (x, y, z)=\left(\Omega^{-1}(\Omega-\Upsilon)(\Omega+\Upsilon)^{-1} \Omega \gamma\left(x_{\imath}, y_{l}, z_{\imath}\right)\right. \text {; }
\end{aligned}
$$

we have

$$
\left(\Omega \gamma(x, y, z \gamma \mathrm{x}, \mathrm{y}, \mathrm{z})=\left(\Omega \gamma\left(x, y_{1}, z, \gamma \mathrm{x}, \mathrm{y}, \mathrm{z},\right) ;\right.\right.
$$

and in particular,

If $\Omega$ is a symmetrical matrix, then $\Upsilon$ is an arbitrary skew symmetrical matrix;

If $\Omega$ is a skew symmetrical matrix, then $\Upsilon$ is an arbitrary symmetrical matrix.

C. II. 